\title{
SOLAR-ISS: A new reference spectrum based on SOLAR/SOLSPEC observations ${ }^{\star}$
}

\author{
M. Meftah ${ }^{1}$, L. Damé ${ }^{1}$, D. Bolsée ${ }^{2}$, A. Hauchecorne ${ }^{1}$, N. Pereira ${ }^{2}$, D. Sluse ${ }^{2}$, G. Cessateur ${ }^{2}$, A. Irbah ${ }^{1}$, J. Bureau ${ }^{1}$, \\ M. Weber ${ }^{3}$, K. Bramstedt ${ }^{3}$, T. Hilbig ${ }^{3}$, R. Thiéblemont ${ }^{1}$, M. Marchand ${ }^{1}$, F. Lefèvre ${ }^{1}$, A. Sarkissian ${ }^{1}$, and S. Bekki ${ }^{1}$ \\ ${ }^{1}$ CNRS, LATMOS, Université Paris Saclay, Université Pierre et Marie Curie, UVSQ, INSU, IPSL, 75005 Paris, France \\ e-mail: Mustapha.Meftah@latmos.ipsl.fr \\ 2 Royal Belgian Institute for Space Aeronomy (BIRA-IASB), 1180 Brussels, Belgium \\ 3 Institute of Environmental Physics, University of Bremen, 28359 Bremen, Germany
}

Received 6 June 2017 / Accepted 18 October 2017

\begin{abstract}
Context. Since April 5, 2008 and up to February 15, 2017, the SOLar SPECtrometer (SOLSPEC) instrument of the SOLAR payload on board the International Space Station (ISS) has performed accurate measurements of solar spectral irradiance (SSI) from the middle ultraviolet to the infrared ( 165 to $3088 \mathrm{~nm}$ ). These measurements are of primary importance for a better understanding of solar physics and the impact of solar variability on climate. In particular, a new reference solar spectrum (SOLAR-ISS) is established in April 2008 during the solar minima of cycles 23-24 thanks to revised engineering corrections, improved calibrations, and advanced procedures to account for thermal and aging corrections of the SOLAR/SOLSPEC instrument.

Aims. The main objective of this article is to present a new high-resolution solar spectrum with a mean absolute uncertainty of $1.26 \%$ at $1 \sigma$ from 165 to $3000 \mathrm{~nm}$. This solar spectrum is based on solar observations of the SOLAR/SOLSPEC space-based instrument.

Methods. The SOLAR/SOLSPEC instrument consists of three separate double monochromators that use concave holographic gratings to cover the middle ultraviolet (UV), visible (VIS), and infrared (IR) domains. Our best ultraviolet, visible, and infrared spectra are merged into a single absolute solar spectrum covering the $165-3000 \mathrm{~nm}$ domain. The resulting solar spectrum has a spectral resolution varying between 0.6 and $9.5 \mathrm{~nm}$ in the $165-3000 \mathrm{~nm}$ wavelength range. We build a new solar reference spectrum (SOLAR-ISS) by constraining existing high-resolution spectra to SOLAR/SOLSPEC observed spectrum. For that purpose, we account for the difference of resolution between the two spectra using the SOLAR/SOLSPEC instrumental slit functions.

Results. Using SOLAR/SOLSPEC data, a new solar spectrum covering the $165-3000 \mathrm{~nm}$ wavelength range is built and is representative of the 2008 solar minimum. It has a resolution better than $0.1 \mathrm{~nm}$ below $1000 \mathrm{~nm}$ and $1 \mathrm{~nm}$ in the $1000-3000 \mathrm{~nm}$ wavelength range. The new solar spectrum (SOLAR-ISS) highlights significant differences with previous solar reference spectra and with solar spectra based on models. The integral of the SOLAR-ISS solar spectrum yields a total solar irradiance of $1372.3 \pm 16.9 \mathrm{Wm}^{-2}$ at $1 \sigma$, that is yet $11 \mathrm{Wm}^{-2}$ over the value recommended by the International Astronomical Union in 2015.
\end{abstract}

Key words. Sun: fundamental parameters - Sun: general

\section{Introduction}

The Sun is a fundamental source of energy for the climate system of the Earth and represents the major natural forcing of the climate on Earth. Total and solar spectral irradiance compose some of the Global Climate Observing System (GCOS) Essential Climate Variables (ECVs) datasets, which provide the empirical evidence needed to understand and predict the evolution of climate ${ }^{1}$. Indeed, total solar irradiance (TSI) and solar spectral irradiance (SSI) measurements and their time series are required to support the work of the United Nations Framework Convention on Climate Change (UNFCCC) and the Intergovernmental Panel on Climate Change (IPCC). Consequently, the absolute determination of the solar spectrum at high resolution is of primary importance. The absolute solar spectrum between 165 and $3000 \mathrm{~nm}$ has many interests ranging from solar physics to atmospheric and climate physics. The solar spectrum is a

\footnotetext{
$\star$ The spectrum shown in Fig. B.1 is available at the CDS via anonymous ftp to cdsarc.u-strasbg. fr (130.79.128.5) or via http://cdsarc.u-strasbg.fr/viz-bin/qcat?J/A+A/611/A1 1 https://public.wmo.int/en/programmes/ global-climate-observing-system
}

key input in radiative transfer and balance in the various layers of the atmosphere of the Earth and other planets. The integral of the solar spectrum measured outside the atmosphere of the Earth equates to the integral of a blackbody spectrum at 5772 K (Planck's law). Spectral shapes of the solar spectrum are very different, especially in the ultraviolet. The solar spectrum consists of a continuum with numerous of dark absorption lines superposed (Fraunhofer lines). A complete characterization of the solar spectrum is not possible from the ground. The atmosphere of the Earth absorbs and scatters large parts of the incoming solar radiation dependent on wavelength. For instance, there is a complete extinction of solar ultraviolet radiation (wavelength shorter than about $300 \mathrm{~nm}$ ) because of ozone and molecular oxygen absorption in the middle atmosphere. The first successful attempt to measure the UV spectrum (from 220 to $290 \mathrm{~nm}$ ) was performed at high altitude above the ozone layer (between 35 and $75 \mathrm{~km}$ ) as described by Durand et al. (1949). These authors used a vacuum grating spectrograph designed at the Naval Research Laboratory (NRL) and installed in the tail fin of a V-2 rocket. During the 1950s, rapid progress was made in solar physics and instrumental technology using rocket and balloon flights. This opened up the stage for space-based 
measurements and, in particular, for obtaining full solar spectra without being hampered by atmospheric absorption. From the 1960 s, measurements in the UV began with the Orbiting Solar Observatory (OSO) program. From the 1970s, regular solar measurements in the UV below $400 \mathrm{~nm}$ were performed by the sensors of the Atmosphere Explorer (AE) satellites and a series of operational remote sensors on National Oceanic and Atmospheric Administration (NOAA) weather satellites. An overview of the main satellite missions that have performed solar spectral irradiance (SSI) observations at wavelengths higher than $100 \mathrm{~nm}$ is given by Ermolli et al. (2013). Space-based monitoring of the solar spectrum over a broad wavelength range (UV, visible, and infrared) started with the SCanning Imaging Absorption spectroMeter for Atmospheric CHartographY (SCIAMACHY; 240-2400 nm) instrument on board the Environment Satellite (Bovensmann et al. 1999) in 2002. There are also measurements obtained by the SOLar STelar Irradiance Comparison Experiment (SOLSTICE; 115-320 nm) and the Spectral Irradiance Monitor (SIM; 300-2400 nm) instruments onboard the Solar Radiation and Climate Experiment (SORCE) satellite (Woods et al. 2000; Rottman 2005) started in 2003, and the SOLar SPECtrometer (SOLSPEC; 165-3088 nm) instrument of the SOLAR monitoring observatory payload (Schmidtke et al. 2006) on board the International Space Station (ISS) started in 2008. One of the objectives of these space-based missions is the quasi-continuous measurement of the solar spectral irradiance variation with the highest possible accuracy. Knowledge of the absolute solar spectral irradiance is necessary for solar physics, atmospheric physics, climate physics, and other fields of physics. Since then, reference solar spectra with high accuracy are required in a variety of disciplines. Owing to current experimental limitations, the published reference solar spectra are rather composites of space-based measurements, ground-based measurements, and solar models data. The following spectra are generally considered as solar reference data:

- The Labs \& Neckel (1968) solar spectrum from $200 \mathrm{~nm}$ to $100 \mu \mathrm{m}$.

- The Peyturaux (1968) solar spectral irradiance from 447.7 to $863.8 \mathrm{~nm}$.

- The Arvesen 1969 solar spectrum (Arvesen et al. 1969) from 205 to $2495 \mathrm{~nm}$.

- The Thekaekara 1973 solar spectrum (Thekaekara 1974) from $115 \mathrm{~nm}$ to $400 \mu \mathrm{m}$.

- The NASA CV-990 reference solar spectrum (Colina et al. 1996) from 119.5 to $2500 \mathrm{~nm}$.

- The ATLAS 3 reference solar spectrum (Thuillier et al. 2003) from 0.5 to $2397.51 \mathrm{~nm}$.

- The Solar Irradiance Reference Spectra (SIRS) for the 2008 Whole Heliosphere Interval (WHI) from 0.1 to $2400 \mathrm{~nm}$ using a combination of satellite and sounding rocket observations (Woods et al. 2009).

- The SCIAMACHY solar spectrum (Pagaran et al. 2011; Hilbig et al. 2017) from 235 to $2384 \mathrm{~nm}$.

The aim of this article is to present a new reference solar spectrum (SOLAR-ISS) from 165 to $3000 \mathrm{~nm}$ (Meftah et al. 2016b) based on recent observations of the SOLAR/SOLSPEC spectrometer on board the International Space Station (Thuillier et al. 2009; Bolsée 2012). The SOLAR/SOLSPEC instrument has a rather low spectral resolution and cannot sufficiently resolve many Fraunhofer lines. The entrance, intermediate, and exit slits of SOLAR/SOLSPEC determine the spectral resolution (or bandpass) of the spectrometer (commonly called the slit function). The SOLAR/SOLSPEC instrument is composed of three channels (UV, VIS and IR) that together cover the wavelengths domain extending from 165 to $\sim 3000 \mathrm{~nm}$. The SOLAR/ SOLSPEC slit functions were measured during ground-based calibration to determine the spectral resolution of the spectrometer. The resulting spectrum has a resolution extending from 0.6 to $9.5 \mathrm{~nm}$. The new reference solar spectrum (SOLAR-ISS) is developed by combining high spectral resolution from reference solar spectra with the SOLAR/SOLSPEC measured spectrum at lower spectral resolution but higher spectral irradiance accuracy. Therefore, we scale a high-resolution spectrum to our SOLAR/SOLSPEC data. Up to $2400 \mathrm{~nm}$, we choose the ATLAS 3 reference solar spectrum (resolution of $0.05 \mathrm{~nm}$ up to $400 \mathrm{~nm}$ ). Above $2400 \mathrm{~nm}$, we choose the new version of the Solar Radiation Physical Modeling (SRPM) theoretical model (with a resolution of $1 \mathrm{~nm}$ above $2400 \mathrm{~nm}$ ) from Fontenla et al. (2015). The new reference solar spectrum is established for the 2008 solar minimum of the solar cycle 24. Comparisons between the new reference solar spectrum and recent reference spectra and models are made. This paper will only deal with solar observations that include the full spectral range from UV to IR. In this paper, no solar variation is provided from the SOLAR/SOLSPEC observations.

\section{SOLAR/SOLSPEC design and background}

SOLAR/SOLSPEC is a space-based qualified spectroradiometer (dimension of $436 \times 437 \times 455 \mathrm{~mm}$, mass of $\sim 29 \mathrm{~kg}$ and nominal power of $\sim 50 \mathrm{~W}$ ) measuring the solar spectral irradiance from 165 to $3088 \mathrm{~nm}$. This instrument, developed under a fruitful CNRS-LATMOS/BIRA-IASB collaboration, was part of the SOLAR payload, externally mounted on board the ISS Columbus module. Since April 5, 2008 and up to February 15, 2017, SOLAR/SOLSPEC performed accurate measurements of solar spectral irradiance from the middle ultraviolet to the infrared. SOLAR/SOLSPEC is an upgraded version of the instruments that have already accomplished several orbital flights without failure. The previous SOLSPEC instruments flew on several ESA and NASA space missions in the 1980s and 1990s and provided the well-known ATLAS 3 solar spectrum composite (Thuillier et al. 2003). The ATLAS 3 composite covering the wavelength range from 0.5 to $\sim 2400 \mathrm{~nm}$ was built using rocket data from $0.5 \mathrm{~nm}$ to Ly- $\alpha$ (Woods et al. 1998), Upper Atmosphere Research Satellite (UARS) data from Ly- $\alpha$ to $200 \mathrm{~nm}$ (Solar Ultraviolet Spectral Irradiance Monitor; SUSIM and SOLar STellar Irradiance Comparison Experiment; SOLSTICE), ATmospheric Laboratory for Applications and Science (ATLAS) data from 200 to $400 \mathrm{~nm}$ (Shuttle Solar Backscatter UltraViolet spectrometer (SSBUV), SUSIM, and SOLSPEC), and ATLAS and EUropean REtrievable CArrier (EURECA) data from 400 to $2400 \mathrm{~nm}$ (SOLSPEC and SOlar SPectrum instrument (SOSP)). As a reminder, the SOLSPEC type instrument has participated in six space-based missions during the last $30 \mathrm{yr}$ (SPACELAB 1, ATLAS 1, 2, and 3, EURECA and SOLAR). One of the main scientific goal of SOLAR/SOLSPEC is to provide a new reference solar spectrum obtained continuously from 165 to $\sim 3000 \mathrm{~nm}$ with a single instrument with three separated double monochromators each with its own optics and detector. This represents a unique performance in space.

The SOLAR/SOLSPEC space-based spectrometer consists of three channels (the UV channel from 165 to $371 \mathrm{~nm}$ (Bolsée et al. 2017), the VIS channel from 285 to $908 \mathrm{~nm}$, and the IR channel from 646 to $3088 \mathrm{~nm}$ ), which cover the wavelength domains extending from 165 to $3088 \mathrm{~nm}$. Each channel 


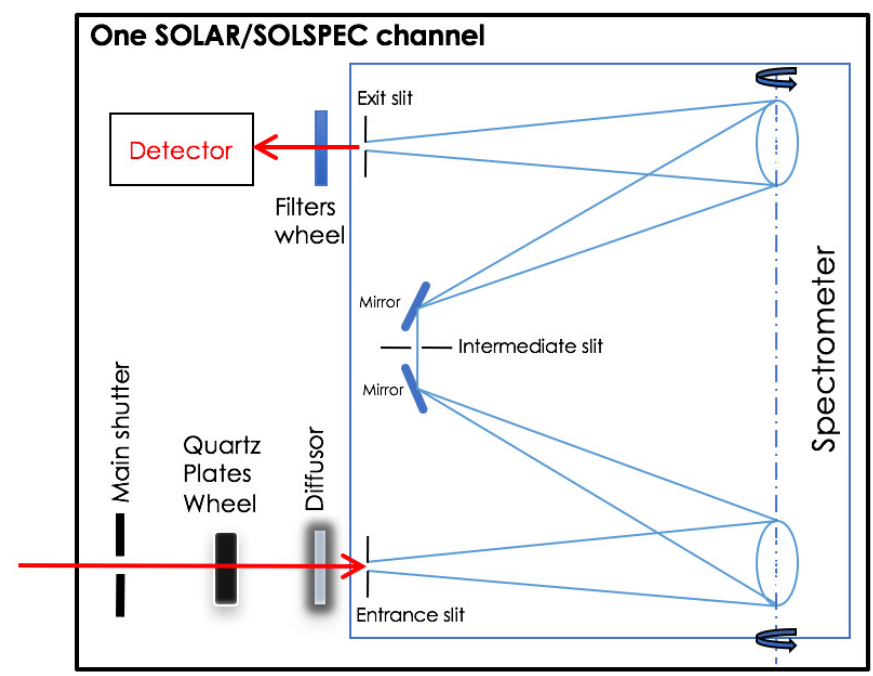

\section{SOLAR/SOLSPEC Instrument}

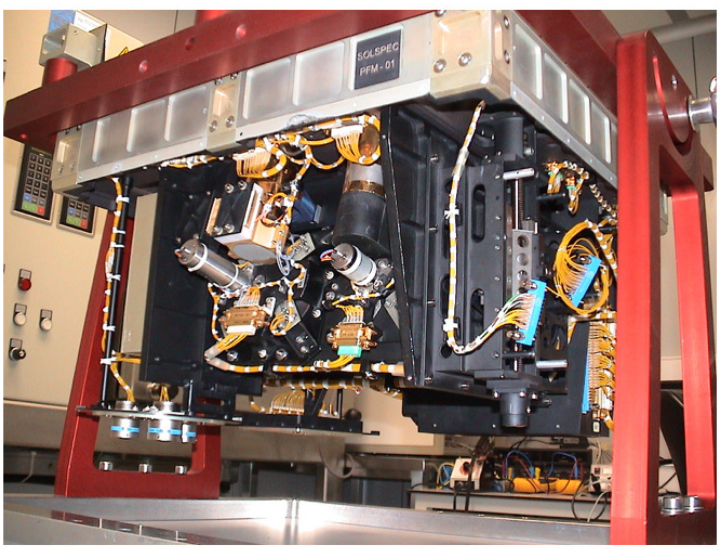

Fig. 1. Left: SOLAR/SOLSPEC optical path of one spectral channel (double-monochromator). The sunlight passes through the entrance slit of the double monochromator and goes in the plane of an intermediate slit through a first monochromator grating and a first mirror. Then, the sunlight goes in the plane of the exit slit through a second mirror and a second monochromator grating identical to the first. Diffraction gratings are used to disperse light (spatial separation of various wavelengths). The wavelength depends on the rotation angle of the grating. The two grating arrangements share the same axis of rotation. This guarantees short scan times at high stability and wavelength accuracy. This design is used in applications in which scattered light must be kept to a minimum. Right: general view of the SOLAR/SOLSPEC flight model with its three channels during integration. SOLAR/SOLSPEC had 3 double monochromators (UV, VIS and IR).

of the SOLAR/SOLSPEC instrument is associated with a double monochromator, which uses concave holographic gratings to minimize the scattered light while maintaining a high throughput. The gratings are mounted on a common mechanical shaft, which rotates by steps and is driven by a nut-screw system. The SOLAR/SOLSPEC sampling interval is less than $0.4 \mathrm{~nm}$ in the UV channel, close to $1 \mathrm{~nm}$ in the VIS channel and close to $4 \mathrm{~nm}$ in the IR channel for common operations but the sampling can be higher. The detectors are photomultiplier tubes in the UV and VIS bands, and a photoconductive detector thermally controlled at $-21.8^{\circ} \mathrm{C}$ with a Peltier thermoelectric cooler in the IR band. Between each exit slit and detector, a wheel carrying second order filters and appropriate attenuators allows the reduction of the incident flux to avoid the saturation of the detectors. The three wheels are positioned as a function of wavelength to take into account the change in solar irradiance and the variation of the spectrometer's responsivity with wavelength, in particular, at both ends of each spectrometer wavelength domain. Diffusors are placed in front of the entrance slit to ensure full illumination of the first grating and also to reduce the effect of pointing uncertainties during observations. The SOLAR/SOLSPEC instrument also contains seven lamps for checking the responsivity and spectral characteristics of the three channels (Bolsée 2012), thus allowing periodical verification to be made on ground and in orbit. The deuterium lamp (nominal or spare) allows a relative calibration of the UV channel. Similarly, the tungsten ribbon lamp (nominal or spare) allows a relative calibration of the VIS channel. Two tungsten ribbon lamps (nominal and redundant) are associated with the IR channel. A hollow cathode lamp verifies spectral resolutions and wavelength scales for the UV and VIS channels. The main optical path of one SOLAR/SOLSPEC channel is illustrated in Fig. 1. The SOLAR/SOLSPEC instrument was not designed with redundant channels.

The radiometric responsivity of each SOLAR/SOLSPEC channel was determined during a set of ground-based calibration campaigns. The instrument was calibrated at PhysikalischTechnische Bundesanstalt (PTB, Braunschweig in Germany) before launch in 2008. The pre-flight absolute calibration of each SOLAR/SOLSPEC channel was carried out with the BB 3200pg blackbody of PTB (Sapritsky et al. 1997; Sperfeld et al. 1998, 2000, 2013). In-flight, regular acquisition with lamps system were performed to track the effects of instrument aging.

\section{Methods for obtaining a new solar spectrum}

In this section, we describe the general methods used to obtain a new reference solar spectrum at high resolution (SOLAR-ISS) based on SOLAR/SOLSPEC observations obtained at lower spectral resolution.

\subsection{Determination of the SOLAR/SOLSPEC spectrum (SS ISOLSPEC $(\lambda))$}

The SOLAR/SOLSPEC measured spectrum in the $165-3000 \mathrm{~nm}$ range is obtained from several spectra related to the three spectrometer channels, i.e.,

- A solar spectrum from 165 to $356 \mathrm{~nm}$ obtained with the UV channel (Meftah et al. 2016a).

- A solar spectrum from 356 to $400 \mathrm{~nm}$ obtained with the VIS channel (Meftah et al. 2016a) and another one from 400 to $656 \mathrm{~nm}$ obtained also with the VIS channel (Bolsée 2012).

- A solar spectrum from 656 to $3000 \mathrm{~nm}$ obtained with the IR channel (Meftah et al. 2017).

From SOLAR/SOLSPEC data and instrumental equations described below, we obtained a new solar spectrum $\left(\operatorname{SSI}_{\operatorname{SOLSPEC}}(\lambda)\right)$ at low spectral resolution between 165 and $3000 \mathrm{~nm}$. This solar spectrum is given at a distance of one astronomical unit. Figure 2 (left) shows the solar spectral irradiance for direct light at the top of the atmosphere of the Earth. For each wavelength, the absolute uncertainty $(1 \sigma)$ of this solar spectrum is given in Fig. 2 (right). 

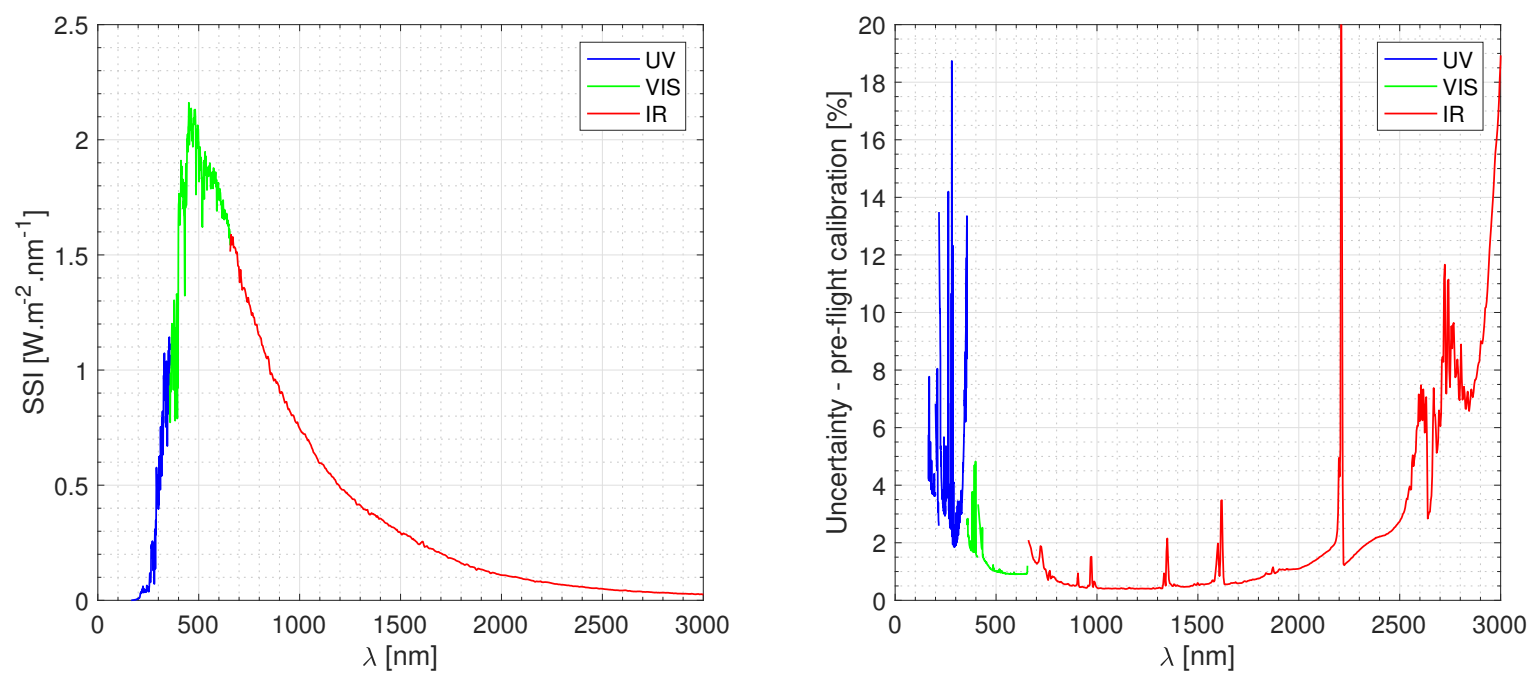

Fig. 2. Left: absolute solar spectral irradiance from 165 to $3000 \mathrm{~nm}$ obtained with the SOLAR/SOLSPEC spectrometer. Wavelength-dependent power input to the top of the atmosphere of the Earth at a standard distance of one astronomical unit from the Sun is plotted Each part of the solar spectrum obtained from a SOLAR/SOLSPEC channel is identified by color. Right: absolute uncertainty of the SOLAR/SOLSPEC spectrum. The absolute radiometric scales have been established during ground-based calibrations (preflight calibration with the PTB blackbody). The absolute accuracy indicates a mean absolute uncertainty of $1.26 \%$ of the total solar irradiance in the $165-3000 \mathrm{~nm}$ range.

\subsubsection{SOLAR/SOLSPEC SSI equation for the UV channel}

The detector of the UV channel is a solar blind EMR photomultiplier tube. For the UV channel, the solar spectral irradiance of the Sun as a function of wavelength $(\lambda)$ and time $(t)$ is given by Eq. (1), i.e.,

$\operatorname{SSI}_{\mathrm{UV}}(\lambda, t)=\frac{\left(S_{n}(\lambda, t)-\frac{<\mathrm{DC}(t)>}{\tau(t)}\right) \times \frac{R_{\mathrm{UV}}(\lambda)}{\operatorname{Deg}_{\mathrm{UV}}(\lambda, t)}}{1-\frac{\alpha_{T}(\lambda, t)}{100} \times\left(T_{P}-T_{S}(t)\right)} \times\left(\frac{z(t)}{1 \mathrm{au}}\right)^{2}$,

where $\operatorname{SSI}_{\mathrm{UV}}(\lambda, t)$ is the solar spectral irradiance measured by SOLAR/SOLSPEC with the UV channel; $S_{n}(\lambda, t)\left(\right.$ counts s $\left.^{-1}\right)$ is the linearized signal provided by the UV detector after the nonlinearity correction. $\langle\mathrm{DC}(t)\rangle$ (counts) is the mean dark counts of the UV detector taken before and after a solar spectrum measurement for a given integration time $(\tau(\mathrm{t})$ in $\mathrm{s}) ; R_{\mathrm{UV}}(\lambda)$ $\left(\mathrm{mWm}^{-2} \mathrm{~nm}^{-1}\right.$ counts $\left.{ }^{-1} \mathrm{~s}\right)$ represents the absolute responsivity of the SOLAR/SOLSPEC UV channel. The SOLAR/SOLSPEC instrumental equation of the UV channel is established to account for thermal and aging corrections. The value $\alpha_{T}(\lambda, t)$ is the temperature coefficient of the UV photomultiplier $\left(\%{ }^{\circ} \mathrm{C}^{-1}\right)$; $T_{P}$ represents the reference temperature of the UV photomultiplier during ground-based calibration $\left(23.3 \pm 1.5^{\circ} \mathrm{C}\right) ; T_{S}(t)\left({ }^{\circ} \mathrm{C}\right)$ represents the temperature of the UV photomultiplier in space; $\operatorname{Deg}_{\mathrm{UV}}(\lambda, t)$ characterizes the degradation of the instrument (dimensionless factor); and $z(t)(\mathrm{km})$ is the distance between SOLAR/SOLSPEC and the Sun. The astronomical unit $(1 \mathrm{au})$ is equal to $149597870.7 \mathrm{~km}$.

\subsubsection{SOLAR/SOLSPEC SSI equation for the VIS channel}

The detector of the VIS channel is a tri-alkali EMR photomultiplier tube. The SOLAR/SOLSPEC instrumental equations are the same for the UV and VIS channels. The similar equation holds for the VIS channel with appropriate substitution in Eq. (1).

\subsubsection{SOLAR/SOLSPEC SSI equation for the IR channel}

The detector of the IR channel is a P2682 PbS photodiode associated with a Peltier thermoelectric cooler. The SOLAR/SOLSPEC instrumental equation of the IR channel is given by Eq. (2), i.e.,

$\operatorname{SSI}_{\mathrm{IR}}(\lambda, t)=\frac{S_{k}(\lambda, t) \times \frac{R_{\mathrm{IR}}(\lambda)}{\delta_{\mathrm{IR}}(\lambda, t)}}{1-k_{\lambda}^{\prime} \times\left(T_{P}-T_{S}(t)\right)} \times\left(\frac{z(t)}{1 \mathrm{au}}\right)^{2}$,

where $\operatorname{SSI}_{\mathrm{IR}}(\lambda, t)$ is the corrected solar spectral irradiance at a given wavelength $(\lambda)$ and for a given time $(t)$, and $S_{k}(\lambda, t)$ (Volt) is the signal provided by the SOLAR/SOLSPEC IR channel with its photoconductive detector (P2682 PbS IR cell). The $S_{k}(\lambda, t)$ signal is obtained after a dark signal correction. $R_{\mathrm{IR}}(\lambda)$ $\left(\mathrm{mWm}^{-2} \mathrm{~nm}^{-1} \mathrm{~V}^{-1}\right)$ represents the absolute response of the SOLAR/SOLSPEC spectrometer for the IR channel; $\delta_{\mathrm{IR}}(\lambda, t)$ is a unitless term, which represents the degradation signal in relation with the aging of the SOLAR/SOLSPEC IR channel; $k_{\lambda}^{\prime}\left({ }^{\circ} \mathrm{C}^{-1}\right)$ is the temperature coefficient of the IR channel, which represents a parameter of the instrument behavior in flight. The value $T_{S}(t)$ $\left({ }^{\circ} \mathrm{C}\right)$ is the temperature of the SOLAR/SOLSPEC central plate and $T_{P}\left({ }^{\circ} \mathrm{C}\right)$ represents the reference temperature of the IR channel during the ground-based calibration with the PTB blackbody $\left(23.3 \pm 1.5^{\circ} \mathrm{C}\right)$.

\subsubsection{SOLAR/SOLSPEC spectrum $\left(\operatorname{SSI}_{\operatorname{SOLSPEC}}(\lambda)\right)$}

The solar spectra obtained with the UV and VIS channels are based on 2008 solar observations. The solar spectrum obtained with the IR channel is based on 2010-2016 observations (combination of all measurements to obtain a reference spectrum). All measurements of the IR channel were used and corrected for thermal effects based on inflight housekeeping temperatures and instrumental thermal characterization performed during a specific set of orbits in March 2016 (Meftah et al. 2017). Given the low variability in the IR wavelength range during a 11-year solar cycle (SSI variations less than $0.05 \%$ from 656 to $3000 \mathrm{~nm}$ ) 


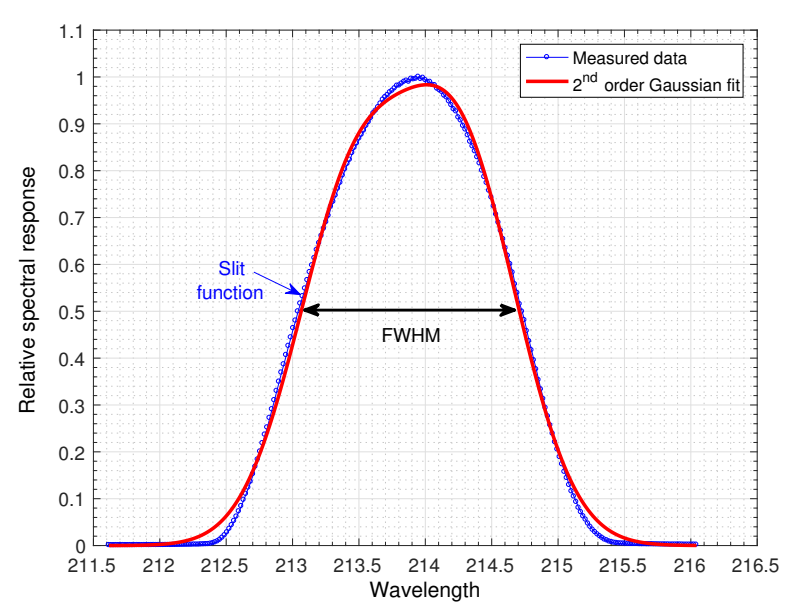

Fig. 3. Relative spectral response (slit function) at $213.8 \mathrm{~nm}$ measured with the SOLAR/SOLSPEC UV channel. A second order Gaussian curve was fit on the experimental data. At $213.8 \mathrm{~nm}$, the bandpass (FWHM) is close to $1.7 \mathrm{~nm}$.

compared to the uncertainties obtained during absolute pre-flight calibration (a minimum uncertainty of $\sim 0.4 \%$ at $1200 \mathrm{~nm}$ ), we can consider the IR absolute solar spectrum as representative of the year 2008. Therefore, the SOLAR/SOLSPEC spectrum resulting from Eq. (3) and shown in Fig. 2 (left) is representative of the 2008 solar minimum of the solar cycle $24 . \operatorname{SSI}_{\text {SOLSPEC }}(\lambda)$ represents the resulting SOLAR/SOLSPEC spectrum (concatenation $(+)$ of the solar spectra obtained with the three spectrometer channels),

$\operatorname{SSI}_{\operatorname{SOLSPEC}}(\lambda)=\operatorname{SSI}_{\mathrm{UV}}(\lambda)+\operatorname{SSI}_{\mathrm{VIS}}(\lambda)+\operatorname{SSI}_{\mathrm{IR}}(\lambda)$.

The spectral resolution depends on the SOLAR/SOLSPEC optical design (Fig. 1) with two main limitations (sampling interval and bandpass during operating mode of the instrument). The sampling interval is the spectral distance between the centers of adjacent spectral wavelengths sampled along the resulting spectrum. From 165 to $356 \mathrm{~nm}$, the sampling interval of the SOLAR/SOLSPEC spectrum is between 0.4 and $0.3 \mathrm{~nm}$. From 356 to $656 \mathrm{~nm}$, the sampling interval is close to $1 \mathrm{~nm}$. From 656 to $3000 \mathrm{~nm}$, the sampling interval is between 4.3 and $3.6 \mathrm{~nm}$. Another important parameter is the bandpass (or resolution). The SOLAR/SOLSPEC slit functions were measured during groundbased calibration to determine the bandpass of each wavelength. The bandpass is defined as the full width at half maximum (FWHM) of the spectral response. The SOLAR/SOLSPEC slit functions are used for convolving the high-resolution solar spectrum before it is scaled to the original SOLAR/SOLSPEC spectrum. The ratio of the SOLAR/SOLSPEC spectrum over the convolved high-resolution external solar spectrum (ATLAS 3/SRPM) is used to scale the latter spectrum but without convolution to obtain the high-resolution SOLAR/SOLSPEC spectrum. The values of the SOLAR/ SOLSPEC slit functions are discussed in the following section.

\subsection{SOLAR/SOLSPEC slit functions $\left(\operatorname{SF}_{\mathrm{SOLSPEC}}(\lambda)\right)$}

The SOLAR/SOLSPEC spectrometer has a finite bandpass (or resolution), which is determined by the sensitivity of the instrument. Therefore, the SOLAR/SOLSPEC instrument provided a smoothed representation of the true solar spectrum. The SOLAR/SOLSPEC slit functions are determined by the design of each channel of the spectrometer. These slit functions were determined experimentally during ground-based calibration, for each channel, using a set of lasers and known emission lines of several spectral lamps ( $\mathrm{Hg}, \mathrm{Zn}, \mathrm{Cd}, \mathrm{He}$, and Ar lamps), thoroughly explained by Bolsée (2012). As these measurements were not carried out in vacuum, the results are limited to $185 \mathrm{~nm}$ for the short wavelengths of the SOLAR/SOLSPEC UV channel. Figure 3 shows, as an example, the slit function at $213.8 \mathrm{~nm}$ (central wavelength) determined using one of the abovementioned spectral lines. The calculation of the FWHM of the slit function provides the bandpass at the corresponding wavelength. For each channel, both the slit function $\left.\operatorname{SF}_{\text {SOLSPEC }}(\lambda)\right)$ and the bandpass are interpolated to a $0.1 \mathrm{~nm}$ grid and shown on the left and right panels of Figs. 4-6 for the UV, VIS and IR channels, respectively. This in-depth knowledge of instrumental slit functions is of great importance for the comparison of solar spectra between various instruments performing in-orbit measurements. The SOLAR/SOLSPEC slit functions have the following characteristics:

- The UV slit functions from 185 to $370 \mathrm{~nm}$ in steps of $0.1 \mathrm{~nm}$ (sampling interval), which are linked with the SOLAR/SOLSPEC spectrum from 165 to $356 \mathrm{~nm}$.

- The VIS slit functions from 312 to $872 \mathrm{~nm}$ in steps of $0.1 \mathrm{~nm}$, which are linked with the SOLAR/SOLSPEC spectrum from 356 to $656 \mathrm{~nm}$.

- The IR slit functions from 594 to $3000 \mathrm{~nm}$ in steps of $0.1 \mathrm{~nm}$, which are linked with the SOLAR/SOLSPEC spectrum from 656 to $3000 \mathrm{~nm}$.

The slit function shown in Fig. 3 is a best case. It is very symmetric. Figures 4-6 illustrate the asymmetries extension of the slit functions. In the SOLAR/SOLSPEC case, the asymmetries of the slits functions (Figs. 4-6) are the results of slight internal misalignments. The SOLAR/SOLSPEC optical design is basically a set of simple subtractive double monochromators that is subject to optical aberrations; we are not using the zero order from a flat mirror linked to the first grating like in a classical Wadsworth design. The slits functions vary as a function of wavelength. The FWHM values of the SOLAR/SOLSPEC slits functions are shown in Figs. 4-6. However, in the data reduction, we use the full profile of the slits functions as shown on the left part of the Figs. 4-6.

\subsection{Sources of high-resolution solar spectra $\left(\operatorname{SSI}_{\mathrm{HR}}(\lambda)\right)$}

We now want to implement in our $\operatorname{SSI}_{\text {SOLSPEC }}(\lambda)$ resulting spectrum the high-resolution information contained in the following solar spectra: the ATLAS 3 solar spectrum (Thuillier et al. 2003) from 165 to $\sim 2400 \mathrm{~nm}$ and the SRPM solar spectrum (Fontenla et al. 2015) from $\sim 2400$ to $3000 \mathrm{~nm}$. The combination of ATLAS 3 and SRPM is called $\operatorname{SSI}_{\mathrm{HR}}(\lambda)$ in the following sections.

\subsection{Determination of the new reference solar spectrum (SOLAR-ISS)}

We base the new SOLAR-ISS spectrum presented in this article on the SOLAR/SOLSPEC absolute calibration $\operatorname{SSI}_{\text {SOLSPEC }}(\lambda)$ ) and the high-resolution information contained in ATLAS 3 and SRPM solar spectra $\left(\operatorname{SSI}_{\mathrm{HR}}(\lambda)\right)$.

The high-resolution solar spectrum $\left(\operatorname{SSI}_{\mathrm{HR}}(\lambda)\right)$ has been convolved $(\otimes)$ with the SOLAR/SOLSPEC slit functions $\left(\operatorname{SF}_{\text {SOLSPEC }}(\lambda)\right)$ as shown in Eq. (4). From Eqs. (5) and (6), we obtained the new SOLAR-ISS spectrum at high spectral 

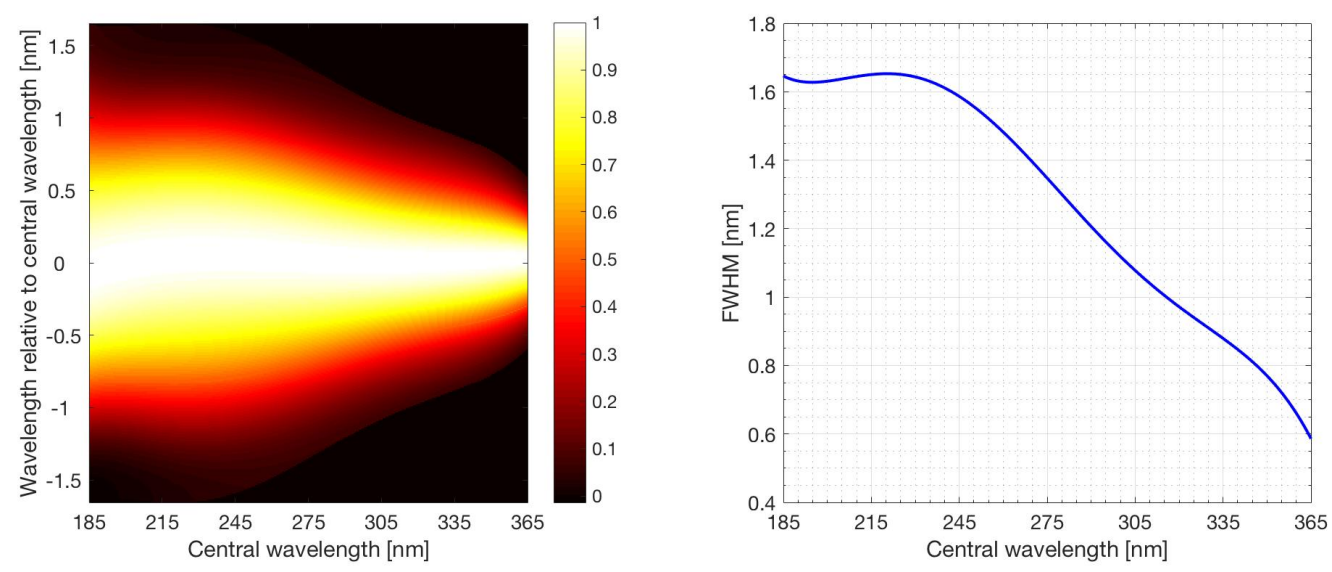

Fig. 4. Left: relative spectral responses distribution in the exit slit of the SOLAR/SOLSPEC UV channel (slit functions). The slit functions of the spectrometer were obtained from ground-based measurements. The point spread function (PSF) profiles are very symmetrical for the UV channel. Right: FWHM values (bandpass or resolution) of the SOLAR/SOLSPEC slit functions (UV channel) are shown.
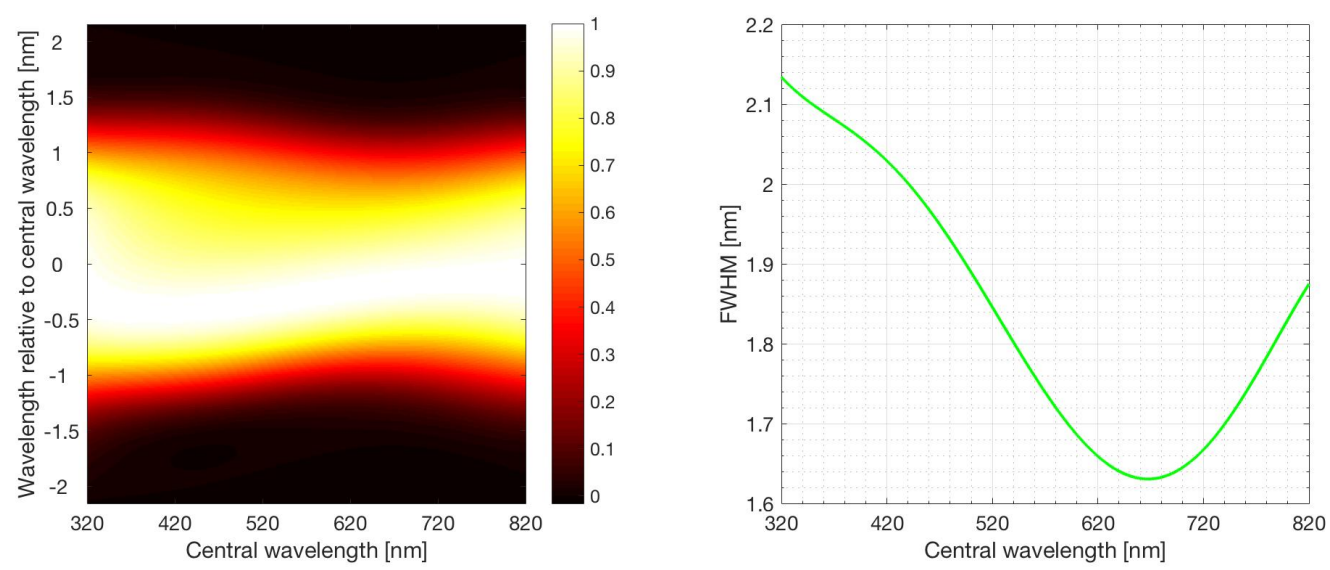

Fig. 5. Left: relative spectral responses distribution in the exit slit of the SOLAR/SOLSPEC VIS channel (slit functions). Right: FWHM values of the SOLAR/SOLSPEC slit functions (VIS channel) are shown.
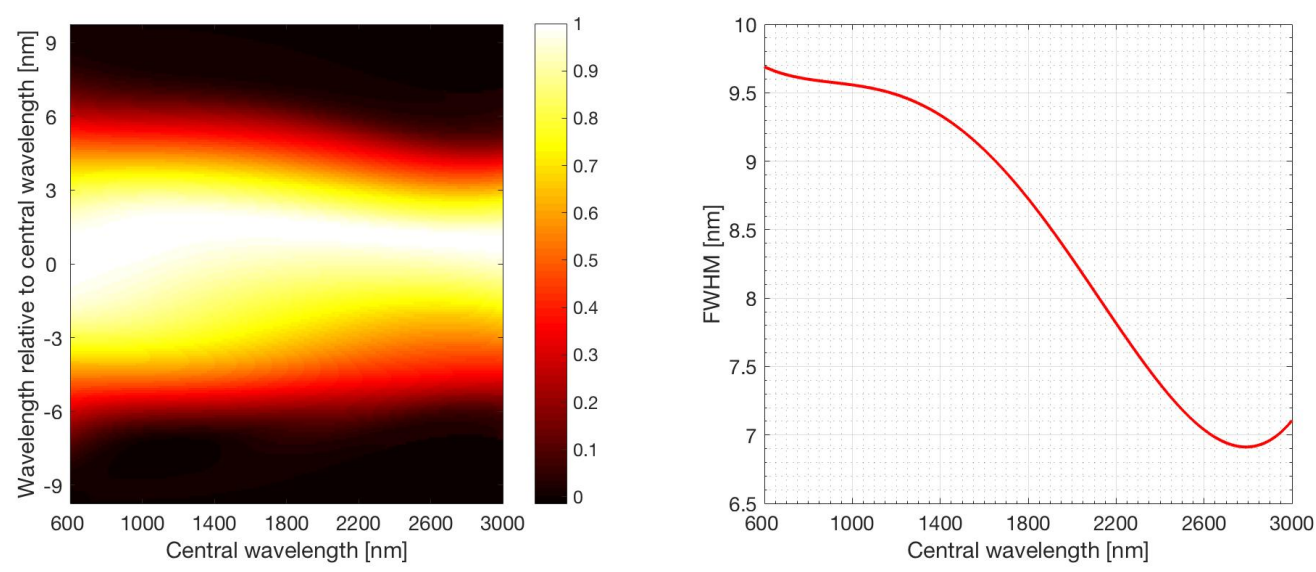

Fig. 6. Left: relative spectral responses distribution in the exit slit of the SOLAR/SOLSPEC IR channel (slit functions). The intensity distributions are not symmetrical. Right: FWHM values of the SOLAR/SOLSPEC slit functions (IR channel) are shown.

resolution between 165 and $3000 \mathrm{~nm}$ at 1 au as follows:

$\operatorname{SSI}_{\mathrm{LR}}(\lambda)=\operatorname{SSI}_{\mathrm{HR}}(\lambda) \otimes \operatorname{SF}_{\mathrm{SOLSPCC}}(\lambda)$

$\operatorname{Ratio}(\lambda)=\frac{\operatorname{SSI}_{\text {SOLSPEC }}(\lambda)}{\operatorname{SSI}_{\mathrm{LR}}(\lambda)}$

$\operatorname{SOLAR}-\operatorname{ISS}(\lambda)=\operatorname{SSI}_{\mathrm{HR}}(\lambda) \times \operatorname{Ratio}(\lambda)$.
The resulting SOLAR/SOLSPEC spectrum $\left(\operatorname{SSI}_{\text {SOLSPEC }}(\lambda)\right.$ ) (4) comes from the combination of the solar spectra based on the three channels of the SOLAR/SOLSPEC instrument given by Eq. (3). The solar spectrum, obtained with the IR channel (from (5) 656 to $3000 \mathrm{~nm}$ ) using the instrumental equation of the IR channel, is more noisy than those obtained with the UV and the VIS (6) channels. This is mainly due to the instrumental correction and 


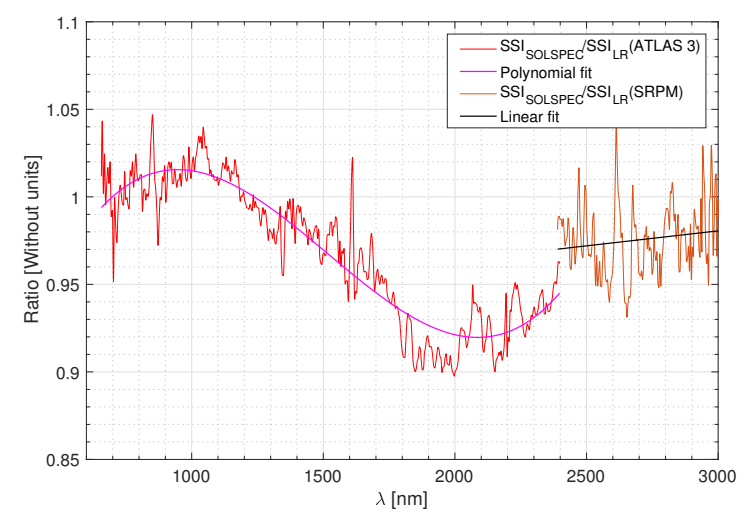

Fig. 7. Ratio $(\lambda)$ between the SOLAR/SOLSPEC spectrum $\left(\operatorname{SSI}_{\text {SOLSPEC }}(\lambda)\right)$ and the ATLAS 3 solar spectrum $\left(\operatorname{SSI}_{\mathrm{LR}}(\lambda)\right)$ between 656 and $2400 \mathrm{~nm}$. The 3-order polynomial fit (magenta curve) is only applied for IR data. Ratio $(\lambda)$ between the SOLAR/SOLSPEC spectrum $\left(\operatorname{SSI}_{\operatorname{SOLSPEC}}(\lambda)\right)$ and the SRPM solar spectrum $\left(\operatorname{SSI}_{\mathrm{LR}}(\lambda)\right)$ between 2400 and $3000 \mathrm{~nm}$ with its linear fit (black curve) is shown.

not to the signal-to-noise ratio $(\mathrm{S} / \mathrm{N})$ of the SOLAR/SOLSPEC IR channel. Therefore, an additional correction is required for the solar spectrum obtained with the SOLAR/SOLSPEC IR channel. Figure 7 shows the ratio between the solar spectrum obtained with the IR channel and the ATLAS 3 solar spectrum in the 656-2400 nm wavelength range. The spikes shown in the plot reveal abrupt changes of transmission in the spectrometer. In order to remove this structures, a third order polynomial fit is used for filtering the ratio given by Eq. (5). The same process is applied between 2400 and $3000 \mathrm{~nm}$ using the SRPM solar spectrum. A linear polynomial fit is applied for the ratio between the SOLAR/SOLSPEC spectrum $\left(\operatorname{SSI}_{\text {SOLSPEC }}(\lambda)\right)$ and the SRPM solar spectrum $\left(\operatorname{SSI}_{\mathrm{LR}}(\lambda)\right)$ between 2400 and $3000 \mathrm{~nm}$.

\section{Results and discussions}

The SOLAR/SOLSPEC instrument has been built to carry out an absolute solar spectrum from 165 to $3000 \mathrm{~nm}$ through an absolute irradiance calibration. With the method described in Sect. 3, we obtained a new solar spectrum at high resolution called "SOLAR-ISS" (Fig. 8 and Appendix B). The new solar spectrum (SOLAR-ISS, Version 1.1) is available in a numerical file online $^{2}$ and at the CDS. The concatenated SOLAR/SOLSPEC data (without the high-resolution adjustment) are also available on demand.

The Sun provides a broad range of power, primarily concentrated around the visible and infrared regions. From the new solar spectrum (SOLAR-ISS), there is $105.8 \mathrm{Wm}^{-2}$ in the ultraviolet region $(165-400 \mathrm{~nm})$. The ultraviolet region extends from 10 to $400 \mathrm{~nm}$; below $165 \mathrm{~nm}$, the solar flux is very weak. Visible light is usually defined as having wavelengths in the range of $400-700 \mathrm{~nm}$. In this region, the flux of the new solar spectrum is $535.5 \mathrm{Wm}^{-2}$. Infrared radiation extends from $700 \mathrm{~nm}$ to $1 \mathrm{~mm}$. In the 700-3000 nm wavelength band, the flux of the new solar spectrum is $703.0 \mathrm{Wm}^{-2}$. The $165-3000 \mathrm{~nm}$ wavelength range then represents $\sim 98 \%$ of the total solar irradiance, which is the total integrated irradiance over the entire solar spectrum. Current accepted TSI values are about 1361-1362 $\mathrm{Wm}^{-2}$ (Kopp \& Lean 2011; Schmutz et al. 2013; Meftah et al. 2014, 2016c), which are representative of the 2008 solar minimum period. The integral

\footnotetext{
2 http://bdap.ipsl.fr/voscat/SOLAR_ISS_V1.html
}

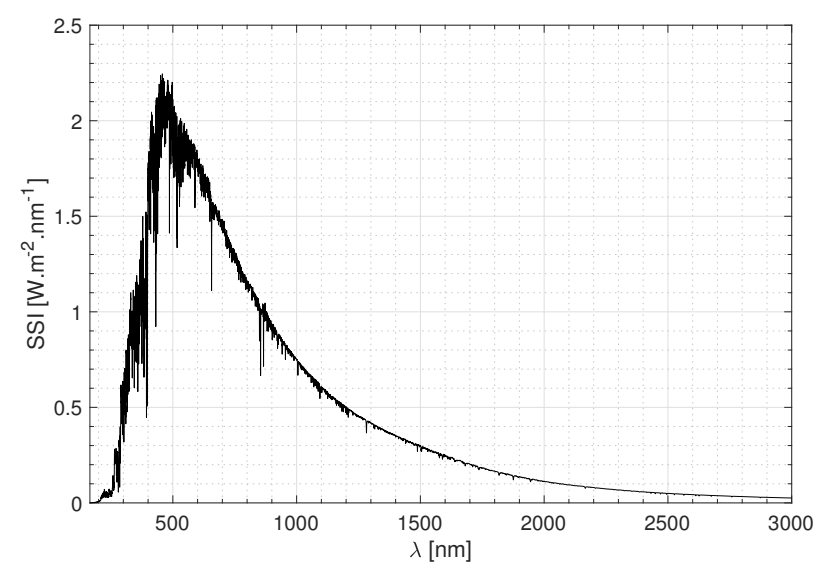

Fig. 8. Solar spectral irradiance obtained with SOLAR/SOLSPEC from 165 to $3000 \mathrm{~nm}$ (SOLAR-ISS at high resolution).

Table 1. Contributions from various wavelength ranges to the total solar irradiance distribution in $\%$ for a blackbody spectrum at $5772 \mathrm{~K}$ (Planck's law) and for the new SOLAR-ISS spectrum.

\begin{tabular}{lllll}
\hline \hline$\Delta \lambda[\mathrm{nm}]$ & $165-400$ & $400-700$ & $700-3000$ & TSI $\left[\mathrm{Wm}^{-2}\right]$ \\
\hline SOLAR-ISS & $7.7 \%$ & $39.0 \%$ & $51.2 \%$ & 1372.3 \\
Blackbody & $12.1 \%$ & $36.5 \%$ & $49.0 \%$ & 1363.6 \\
\hline
\end{tabular}

Notes. From 165 to $3000 \mathrm{~nm}$, the integrated flux of the SOLAR-ISS spectrum is $1344.3 \mathrm{Wm}^{-2}$. Adding the contribution from a solar model $(3000 \mathrm{~nm}$ to $160 \mu \mathrm{m})$, we obtain a TSI of $1372.3 \mathrm{Wm}^{-2}$.

of the SOLAR-ISS spectrum is $1344.3 \pm 16.9 \mathrm{Wm}^{-2}$ at $1 \sigma$ in the $165-3000 \mathrm{~nm}$ range. Adding $28.0 \mathrm{Wm}^{-2}$ for the spectral region $3-160 \mu \mathrm{m}$ as calculated from the Spectral And Total Irradiance REconstruction for the Satellite era (SATIRE-S) semi-empirical model, the TSI from SOLAR-ISS sums up to $1372.3 \mathrm{Wm}^{-2}$. The difference between the accepted and measured TSI value and SOLAR-ISS TSI is around $11 \mathrm{Wm}^{-2}$, which is within the SOLAR-ISS uncertainty $\left( \pm 1.26 \%\right.$ or $\pm 16.9 \mathrm{Wm}^{-2}$ at $\left.1 \sigma\right)$.

Table 1 summarizes the contributions of three wavelengths bands $(165-400 \mathrm{~nm}, 400-700 \mathrm{~nm}$, and $700-3000 \mathrm{~nm})$ to the SOLAR-ISS total solar irradiance and the integrated spectrum of a blackbody at $5772 \mathrm{~K}$.

The accurate determination of a new solar spectrum in the $165-3000 \mathrm{~nm}$ range is challenging. SOLAR-ISS represents a new spectrum. The SOLAR-ISS spectrum (quiet Sun during the 2008 solar minimum) was compared with the ATLAS 3 solar spectrum from 165 to $2397.5 \mathrm{~nm}$. The integral of the ATLAS 3 solar spectrum is $1330.1 \mathrm{Wm}^{-2}$ in the $165-2397.5 \mathrm{~nm}$ band. In the same wavelength range, the integral of the SOLAR-ISS spectrum is $1320.9 \mathrm{Wm}^{-2}$. The integral of the SOLAR-ISS spectrum is closer to recent radiometric TSI determinations. There is a difference of about $9 \mathrm{Wm}^{-2}$, which cannot be justified by differences in the acquisition dates. To better see the differences between the two solar spectra (ATLAS 3 and SOLAR-ISS), we plotted these spectra according to their solar brightness temperatures, which are obtained from Planck's law as shown in Fig. 9. One can notice that there are differences, which depend on the wavelength domain and, particularly, in the infrared band.

When integrating the original complete spectrum of ATLAS 3 , its value appears to be more than $30 \mathrm{Wm}^{-2}$ over the actual TSI value $\left(\sim 1361 \mathrm{Wm}^{-2}\right)$ recommended by the International Astronomical Union in 2015. As a consequence, a uniform 


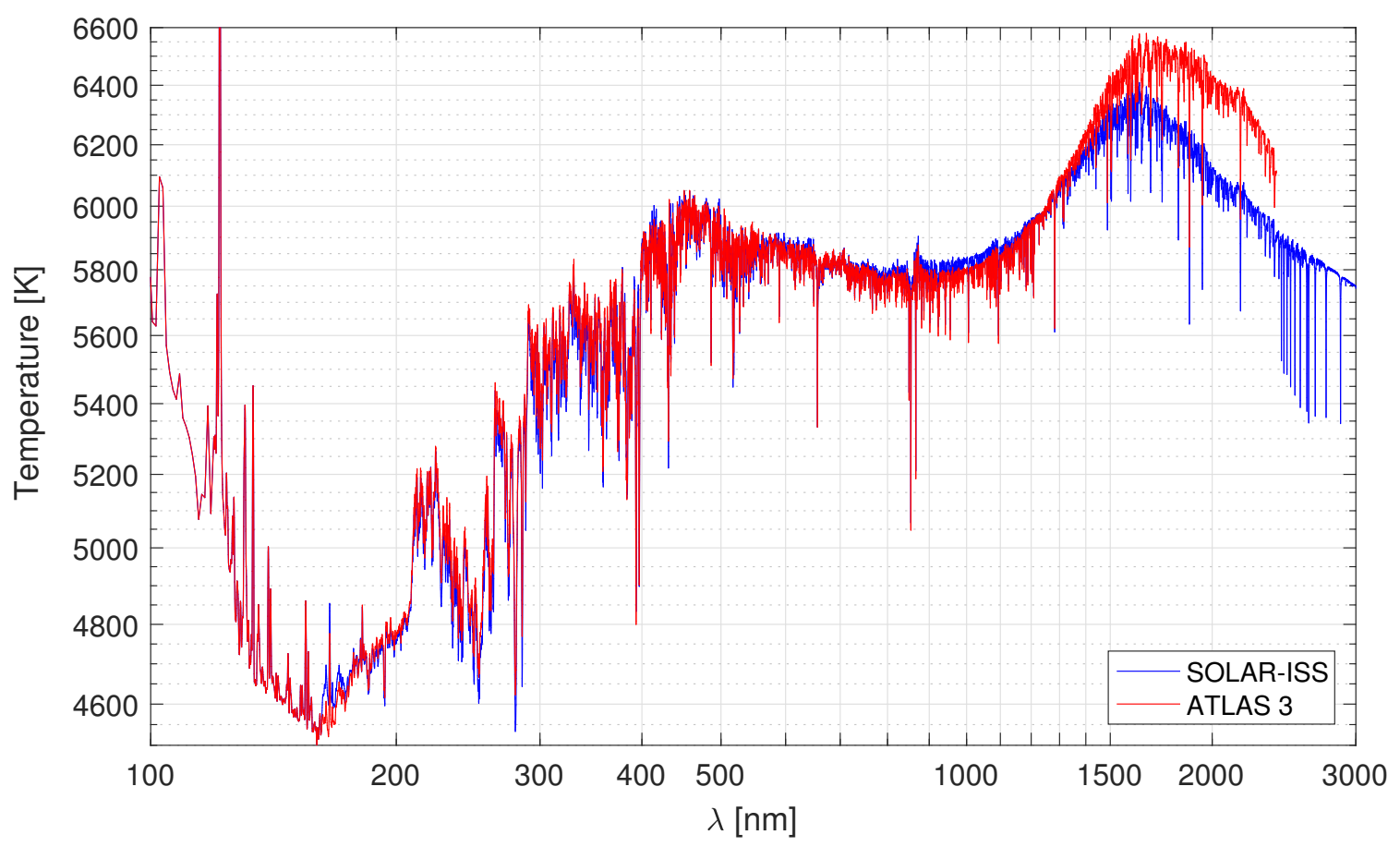

Fig. 9. Brightness temperature of the Sun obtained from the ATLAS 3 solar spectrum (red curve) and SOLAR-ISS data during the 2008 solar minimum (blue curve). From 0.5 to $165 \mathrm{~nm}$, the SOLAR-ISS brightness temperature of the Sun (blue curve) is given by ATLAS 3 data.

reduction of $1.4 \%$ was applied to the ATLAS 3 solar spectrum (with a TSI target of $1367.7 \mathrm{Wm}^{-2}$, which is the previous recommended value) by Thuillier et al. (2003), without any reasonable or justified distribution pattern. In comparison, the integration of SOLAR-ISS yields $1372 \mathrm{Wm}^{-2}$ (yet $11 \mathrm{Wm}^{-2}$ over though). We think that such a standardization approach is not appropriate in ATLAS 3. Our approach (SOLAR-ISS), however, which provides a spectrum obtained with a controlled chain of calibration from PTB up to space, reduces the chances of systematic errors propagated over the whole spectrum. Therefore, the ATLAS 3 solar spectrum is questionable. Moreover, in water vapor bands in the infrared, ATLAS/SOLSPEC calibrations were never carried out under vacuum nor controlled dry atmosphere (Bolsée 2012); along the $0.85 \mathrm{~m}$ distance between the instrument and the Heidelberg blackbody versus the $1.30 \mathrm{~m}$ distance between SOLAR/SOLSPEC and the PTB blackbody errors were introduced up to $6 \%$. Otherwise, ATLAS 3 is limited in the infrared to $2400 \mathrm{~nm}$, while the new SOLAR-ISS reference spectrum extends to $3000 \mathrm{~nm}$ with a traceability of the slits functions of the SOLAR/SOLSPEC instrument.

The SOLAR-ISS spectrum is also compared with reference spectra (Tables 2, 3 and Appendix A) and solar spectra based on models (Tables 2 and 3). We use two reference solar spectra, i.e., ATLAS 3 from Thuillier et al. (2003) and WHI 2008 from Woods et al. (2009), a theoretical model, i.e., the SRPM model from Fontenla et al. (2015) for wavelengths between 101.3 and $10018.3 \mathrm{~nm}$, and two models, i.e., the SATIRE-S from Yeo et al. (2014) and the NRL model from Coddington et al. (2016). The SATIRE-S (Yeo et al. 2014) model is semi-empirical, from $115.5 \mathrm{~nm}$ to $160 \mu \mathrm{m}$, and uses full-disk magnetograms and continuum images of the Sun to quantify the fractional disk area coverage by various surface components (quiet Sun, sunspot umbrae, sunspot penumbrae, faculae, and network) and their spatial distribution. The solar spectral irradiance provided by the Naval Research Laboratory Solar Spectral Irradiance (NRLSSI) model (Lean 2000) is an empirical model, which aims to reconstruct
Table 2. Comparisons between the SOLAR-ISS spectrum and various solar spectra (ATLAS 3, SRPM, WHI 2008, SATIRE-S, and NRLSSI) for various wavelength bands.

\begin{tabular}{llll}
\hline \hline$\Delta \lambda[\mathrm{nm}]$ & $165-400$ & $400-700$ & $700-2400$ \\
\hline SOLAR-ISS/ATLAS 3 & 0.959 & 1.000 & 0.993 \\
SOLAR-ISS/WHI 2008 & 0.999 & 1.012 & 1.007 \\
SOLAR-ISS/SRPM & 0.862 & 0.975 & 1.012 \\
SOLAR-ISS/SATIRE-S & 0.994 & 1.012 & 1.010 \\
SOLAR-ISS/NRLSSI & 0.972 & 1.008 & 1.007 \\
\hline
\end{tabular}

Table 3. Comparisons between the SOLAR-ISS spectrum and different solar spectra between 165 and $2400 \mathrm{~nm}$.

\begin{tabular}{ll}
\hline \hline$\Delta \lambda[\mathrm{nm}]$ & $165-2400$ \\
\hline SOLAR-ISS/ATLAS 3 & 0.993 \\
SOLAR-ISS/WHI 2008 & 1.009 \\
SOLAR-ISS/SRPM & 0.983 \\
SOLAR-ISS/SATIRE-S & 1.009 \\
SOLAR-ISS/NRLSSI & 1.005 \\
\hline
\end{tabular}

long-term SSI from $120 \mathrm{~nm}$ to $100 \mu \mathrm{m}$. This model uses historical estimates of faculae brightening and sunspot darkening to extend in time wavelength-dependent parameterizations of SSI derived from satellite measurements and models. In the ultraviolet band $(165-400 \mathrm{~nm})$, the SOLAR-ISS spectrum is closer to the WHI 2008 solar spectrum (best match). In the visible band (400$700 \mathrm{~nm}$ ), all the spectra studied are identical within $2.5 \%$. In the infrared band (700-2400 nm), only the SOLAR-ISS/ATLAS 3 ratio is smaller than one.

Figure 10 shows the ratios of the SOLAR-ISS solar irradiance to several spectra (ATLAS 3, SRPM, WHI, SATIRE$\mathrm{S}$, and NRLSSI). The most important systematic discrepancies between SOLAR-ISS and all other solar spectra are observed 

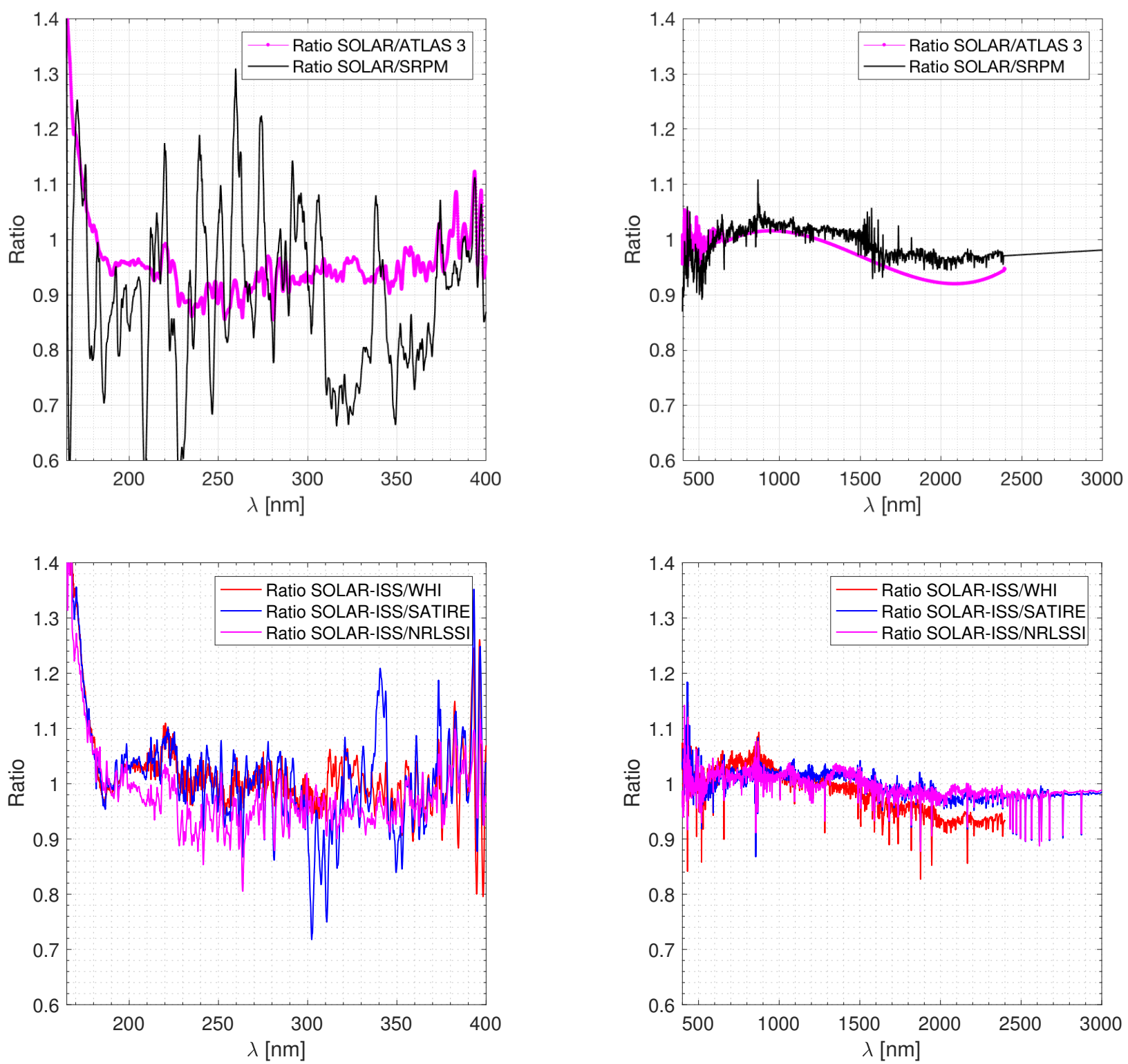

Fig. 10. Top: ratio of the SOLAR-ISS spectrum to ATLAS 3 and SRPM solar spectra. Bottom: ratio of the SOLAR-ISS spectrum to WHI 2008, SATIRE-S, and NRLSSI solar spectra in the $165-3000 \mathrm{~nm}$ wavelength range is shown.

for wavelengths smaller than $180 \mathrm{~nm}$. The largest differences are found between SOLAR-ISS and SRPM in the 200-400 nm wavelength range. Part of these fluctuations may be explained by differences in the instrument profile (i.e., the variation of the instrument response within the coarse resolution element) and wavelength calibration of the instruments (SOLAR/SOLSPEC, other instruments in relation with the dedicated solar reference spectrum) compared to the simple approach used to convolve the SRPM high-resolution solar spectrum. The instrument profile and its variation with wavelength plays an important role, particularly in the middle ultraviolet, in determining the shape of the broad features that represent a very large number of highly packed narrow solar spectral lines. Plots of the spectra are more clear than ratios for the middle ultraviolet. The SRPM solar spectrum shown is given for the quiet-Sun component B, as if the whole Sun was only covered by this, which is close to the quietest state of the Sun, but of course solar activity produces changes that are substantial at the shortest wavelengths. Regarding the
280-410 nm wavelength range, the SRPM spectra calculations in Fontenla et al. (2015) are clearly missing opacity in this range and a revision of the $\mathrm{CH}$ opacity is ongoing (Fontenla 2017, priv. comm.). This may explain a low part of the discrepancies between SRPM and SOLAR-ISS in the $280-410 \mathrm{~nm}$ wavelength range.

In the visible band, the SSI values of the different spectra agree very well. In the infrared band, the discrepancies are very small between SOLAR-ISS and other solar spectra. From $1500 \mathrm{~nm}$, the differences become larger between SOLAR-ISS and other solar reference spectra (ATLAS 3 and WHI 2008) as shown in Fig. 10 and as observed initially by Harder et al. (2010) with the SIM instrument.

An uncertainty of $1 \%$ of the SSI measurements in the 400 $3000 \mathrm{~nm}$ band corresponds to an error of $\sim 12 \mathrm{Wm}^{-2}$ in the TSI. This observation highlights the interest in developing new spacebased instruments to perform very accurate measurements of the solar spectral irradiance in the 400-3000 $\mathrm{nm}$ band (uncertainty less than $0.1 \%$ ). 


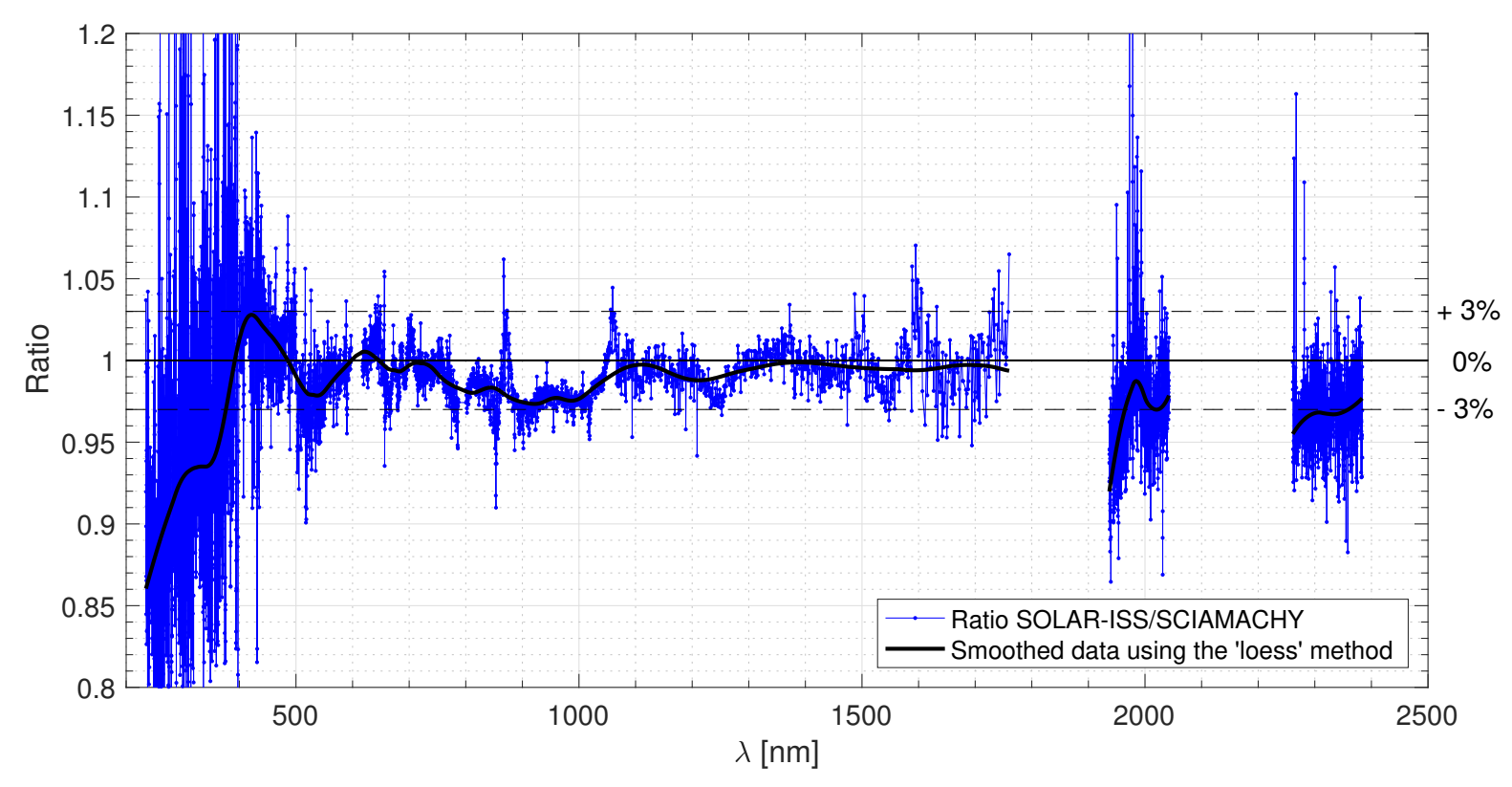

Fig. 11. Ratio of the SOLAR-ISS spectrum to SCIAMACHY solar reference spectrum obtained February 23, 2003 (version 8 revised, preliminary). The comparison between SOLAR-ISS and SCIAMACHY is very good in the visible and in the infrared domains.

Finally, we made a comparison between the SOLAR-ISS spectrum and a very recently updated SCIAMACHY solar reference spectrum (Hilbig et al. 2017) as shown in Fig. 11. In general, the agreement between SCIAMACHY and SOLARISS is good and mostly within 3\%, however, the SOLARISS/SCIAMACHY ratio is lower than 1 in the $235-2384 \mathrm{~nm}$ wavelength range, which means that the integrated flux of the SCIAMACHY spectrum is larger than that of the SOLAR-ISS spectrum. The SCIAMACHY solar spectrum is based on solar measurements of February 27, 2003, which is not representative of solar minimum condition. Therefore, the comparison with SOLAR-ISS is essentially valid in the near UV, visible, and infrared domains. Between 400 and $1760 \mathrm{~nm}$, the SOLARISS/SCIAMACHY ratio is well within $3 \%$, while the SOLARISS/ATLAS 3 ratio can reach $6.5 \%$ at $1760 \mathrm{~nm}$ (Fig. 10, top). This is another indication that the ATLAS 3 solar spectrum in the infrared domain is biassed. Above $1936 \mathrm{~nm}$, the SOLARISS/SCIAMACHY ratio is greater than $3 \%$. To conclude, the SOLAR-ISS spectrum is very close to the SCIAMACHY solar spectrum in the $400-1760 \mathrm{~nm}$ wavelength range.

\section{Conclusions}

The SOLAR/SOLSPEC space-based instrument has measured the solar spectral irradiance from 165 to $3000 \mathrm{~nm}$ at 0.6 to $9.5 \mathrm{~nm}$ spectral resolution during the almost nine years of the SOLAR/ISS mission. The instrument was calibrated using the PTB standard blackbody developed for temperatures up to $3200 \mathrm{~K}$. The absolute accuracy, based on a detailed analysis of error sources, indicates a mean absolute uncertainty of $1.26 \%$ of the total solar irradiance in the $165-3000 \mathrm{~nm}$ range. Using SOLAR/SOLSPEC data, a new solar spectrum (SOLAR-ISS) covering the 165-3000 $\mathrm{nm}$ wavelength range is built with added high spectral resolution. This SOLAR-ISS spectrum is developed by combining high spectral resolution from reference solar spectra with the resulting SOLAR/SOLSPEC spectrum through the knowledge of the slit functions of the SOLAR/SOLSPEC instrument. This new solar spectrum (SOLAR-ISS) highlights differences with previous solar reference spectra (Table A.1) and with solar spectra based on models. They mostly agree but diverge in the infrared. Total and Spectral solar Irradiance Sensor (TSIS) is scheduled to launch to the International Space Station in November 2017 on a SpaceX Falcon 9; TSIS will provide new light on the absolute determination of the solar spectrum, in particular of the infrared part. Today, SOLAR-ISS represents a new reference solar spectrum representative of the 2008 solar minimum. The SOLAR-ISS mission provides an accurate distribution of the power input of the Sun across ultraviolet, visible, and infrared wavelengths of light.

Acknowledgements. The SOLAR/SOLSPEC team acknowledges support from the Centre National de la Recherche Scientifique (CNRS, France), the Centre National d'Études Spatiales (CNES, France), the PROgramme de Développement d'Expériences scientifiques Office (PRODEX Office, Belgium), the Belgian Federal Science Policy Office (BELSPO Office) through the ESA-PRODEX program (contract 4000110593 for 2016-2017), the Solar-Terrestrial Centre of Excellence (STCE, Belgium), the Institute of Environmental Physics University of Bremen, the Bundesministerium für Forschung und Technologie (Germany), and the Laboratory for Atmospheric and Space Physics (LASP, USA). T. Hilbig, K. Bramstedt, and M. Weber acknowledge the support from BMBF via the SCIASOL project as part of the priority program ROMIC (Role of the Middle Atmosphere in Climate). The authors would like to thank Dr. Jerald Harder (LASP), Dr. Martin Snow (LASP), Dr. Juan Fontenla (NorthWest Research Associates), Dr. Julian Gröbner (Physikalisch-Metorologisches Observatorium Davos), Dr. Astrid Orr (ESA), Dr. Alexander Shapiro (Max-Planck-Institut), and Thomas Foujols (LATMOS) for their suggestions and help. The authors would like to thank Dr. Gary Rottman for his assistance in evaluating this paper. The developing institutes of the SOLAR/SOLSPEC space qualified spectro-radiometer are LATMOS (formerly Service d'Aéronomie du CNRS) and BIRA-IASB (Institut d'Aéronomie Spatiale de Belgique).

\section{References}

Arvesen, J. C., Griffin, Jr., R. N., \& Pearson, Jr., B. D. 1969, Appl. Opt., 8, 2215 Bolsée, D. 2012, Ph.D. Thesis, Université libre de Bruxelles, École polytechnique de Bruxelles

Bolsée, D., Pereira, N., Gillotay, D., et al. 2017, A\&A, 600, A21

Bovensmann, H., Burrows, J. P., Buchwitz, M., et al. 1999, J. Atm. Sci., 56, 127

Coddington, O., Lean, J. L., Pilewskie, P., Snow, M., \& Lindholm, D. 2016, Bull. Amer. Meteorol. Soc., 97, 1265 
Colina, L., Bohlin, R. C., \& Castelli, F. 1996, AJ, 112, 307

Durand, E., Oberly, J. J., \& Tousey, R. 1949, ApJ, 109, 1

Ermolli, I., Matthes, K., Dudok de Wit, T., et al. 2013, Atm. Chem. Phys., 13, 3945

Fontenla, J. M., Stancil, P. C., \& Landi, E. 2015, ApJ, 809, 157

Harder, J. W., Thuillier, G., Richard, E. C., et al. 2010, Sol. Phys., 263, 3

Hilbig, T., Weber, M., Bramstedt, K., et al. 2017, Sol. Phys., submitted

Kopp, G., \& Lean, J. L. 2011, Geophys. Res. Lett., 38, 1706

Labs, D., \& Neckel, H. 1968, Z. Astrophys., 69, 1

Lean, J. 2000, Geophys. Res. Lett., 27, 2425

Meftah, M., Dewitte, S., Irbah, A., et al. 2014, Sol. Phys., 289, 1885

Meftah, M., Bolsée, D., Damé, L., et al. 2016a, Sol. Phys., 291, 3527

Meftah, M., Bolsée, D., Hauchecorne, A., et al. 2016b, A new solar reference spectrum obtained with the SOLAR/SOLSPEC space-based spectroradiometer, AGU Fall Meeting 2016

Meftah, M., Chevalier, A., Conscience, C., \& Nevens, S. 2016c, J. Space Weather Space Climate, 6, A34

Meftah, M., Damé, L., Bolsée, D., et al. 2017, Sol. Phys., 292, 101

Pagaran, J., Weber, M., Deland, M. T., Floyd, L. E., \& Burrows, J. P. 2011, Sol. Phys., 272, 159

Peyturaux, R. 1968, Ann. Astrophys., 31, 227

Rottman, G. 2005, Sol. Phys., 230, 7
Sapritsky, V. I., Khlevnoy, B. B., Khromchenko, V. B., et al. 1997, Appl. Opt., 36,5403

Schmidtke, G., Fröhlich, C., \& Thuillier, G. 2006, Adv. Space Res., 37, 255

Schmutz, W., Fehlmann, A., Finsterle, W., Kopp, G., \& Thuillier, G. 2013, AIP Conf. Ser., 1531, 624

Sperfeld, P., Metzdorf, J., Galal Yousef, S., Stock, K. D., \& Möller, W. 1998, Metrologia, 35, 267

Sperfeld, P., Galal Yousef, S., Metzdorf, J., Nawo, B., \& Möller, W. 2000, Metrologia, 37, 373

Sperfeld, P., Pape, S., \& Nevas, S. 2013, in AIP Conf. Ser., 1531, 801

Thekaekara, M. P. 1974, Appl. Opt., 13, 518

Thuillier, G., Hersé, M., Labs, D., et al. 2003, Sol. Phys., 214, 1

Thuillier, G., Foujols, T., Bolsée, D., et al. 2009, Sol. Phys., 257, 185

Woods, T. N., Rottman, G. J., Bailey, S. M., Solomon, S. C., \& Worden, J. R. 1998, Sol. Phys., 177, 133

Woods, T. N., Rottman, G. J., Harder, J. W., et al. 2000, in Earth Observing Systems V, ed. W. L. Barnes, Proc. SPIE, 4135, 192

Woods, T. N., Chamberlin, P. C., Harder, J. W., et al. 2009, Geophys. Res. Lett., 36, L01101

Yeo, K. L., Krivova, N. A., Solanki, S. K., \& Glassmeier, K. H. 2014, A\&A, 570, A85 


\section{Appendix A: Integrated power in specific bands for}

ATLAS 3, WHI 2008, and SOLAR-ISS

Table A.1. Integrated power in specific bands (between 201 and $2423 \mathrm{~nm}$ ) for three solar spectra considered as solar reference data.

\begin{tabular}{llll}
\hline \hline Start integral & \multicolumn{3}{l}{ Stop integral } \\
$\lambda_{1}[\mathrm{~nm}]$ & $\lambda_{2}[\mathrm{~nm}]$ & $I_{0}\left[\mathrm{Wm}^{-2}\right]$ & $I_{0} / 1361$ \\
\hline ATLAS 3 & - & - & - \\
201 & 300 & 15.158 & 0.0111 \\
300 & 400 & 95.017 & 0.0698 \\
400 & 691 & 522.226 & 0.3837 \\
691 & 972 & 303.777 & 0.2232 \\
972 & 1630 & 294.094 & 0.2161 \\
1630 & 2423 & 101.314 & 0.0744 \\
\hline 201 & 2423 & 1331.612 & 0.9784 \\
\hline WHI 2008 & - & - & - \\
201 & 300 & 14.274 & 0.0105 \\
300 & 400 & 92.551 & 0.0680 \\
400 & 691 & 521.042 & 0.3828 \\
691 & 972 & 298.416 & 0.2193 \\
972 & 1630 & 295.755 & 0.2173 \\
1630 & 2423 & 101.125 & 0.0743 \\
\hline 201 & 2423 & 1323.103 & 0.9722 \\
\hline SOLAR-ISS & - & - & - \\
201 & 300 & 14.003 & 0.0103 \\
300 & 400 & 92.077 & 0.0677 \\
400 & 691 & 522.410 & 0.3838 \\
691 & 972 & 306.758 & 0.2254 \\
972 & 1630 & 293.040 & 0.2153 \\
1630 & 2423 & 94.442 & 0.0694 \\
\hline 201 & 2423 & 1322.731 & 0.9719 \\
\hline & & &
\end{tabular}




\section{Appendix B: SOLAR-ISS, a new solar reference spectrum extending to $3000 \mathrm{~nm}$}
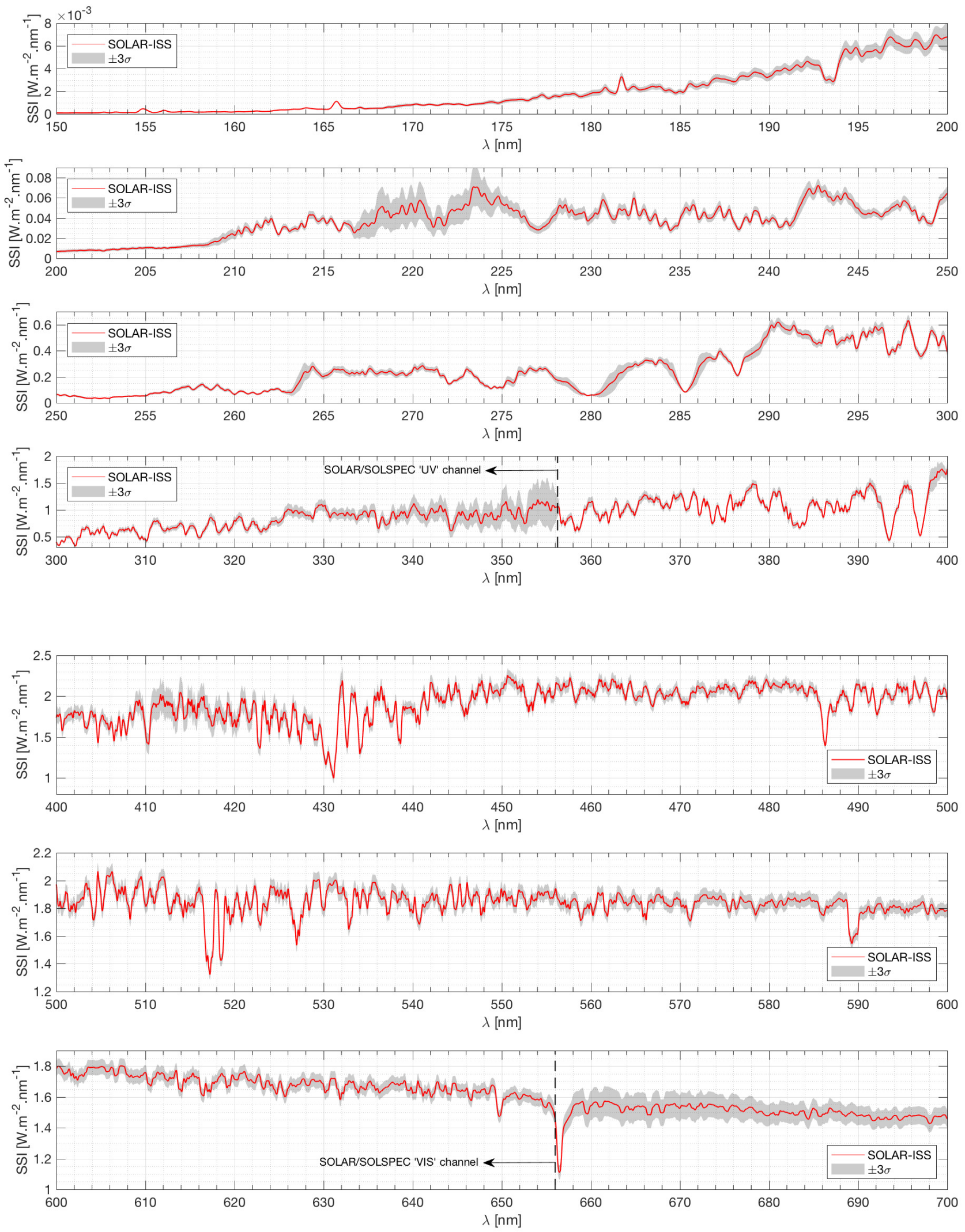

Fig. B.1. SOLAR-ISS spectral irradiance from 150 to $700 \mathrm{~nm}$ at a distance of one astronomical unit (in vacuum conditions) and its uncertainties at $\pm 3 \sigma$. Below $165 \mathrm{~nm}$, no uncertainty value is provided (limit of calibration measurements of the SOLAR/SOLSPEC instrument). 

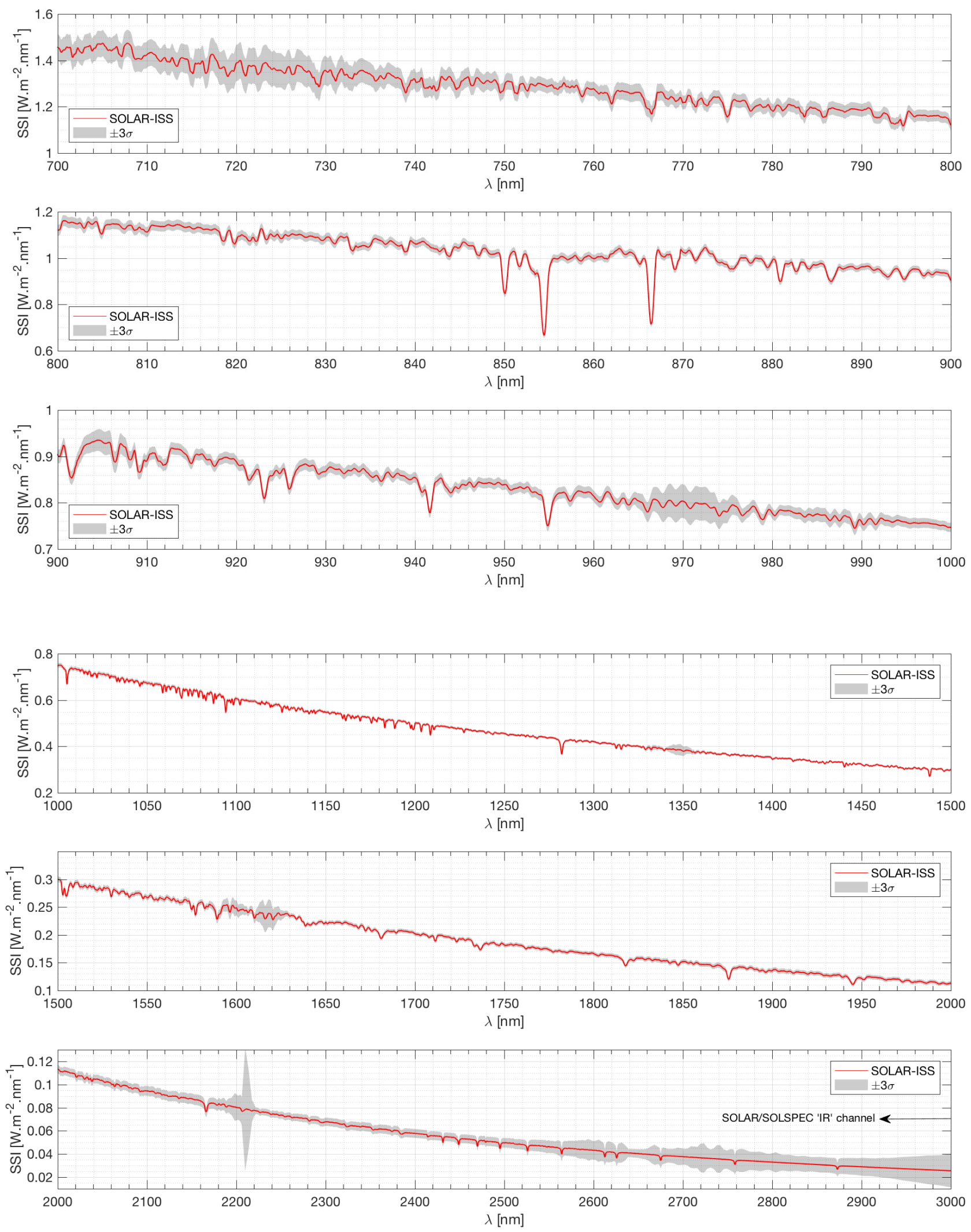

Fig. B.2. SOLAR-ISS spectral irradiance from 700 to $3000 \mathrm{~nm}$ at a distance of one astronomical unit (in vacuum conditions) and its uncertainties at $\pm 3 \sigma$. 